\title{
28 Research Soure \\ Comparison of Visual Performance between Refractive and Diffractive Multifocal Intraocular Lenses
}

\section{Lingying Ye}

Wenzhou Medical University Eye Hospital

\section{Tianyu Chen}

eye hospital of wenzhou medical hospital

\section{Zhixiang $\mathrm{Hu}$}

eye hospital of wenzhou medical university

\section{Qiuwen Yang}

eye hospital of wenzhou medical university

\section{Qiudong Su}

eye hospital of wenzhou medical university

Jin Li ( $\nabla$ yhwang1026@163.com )

eye hospital of wenzhou medical university https://orcid.org/0000-0003-3745-9547

\section{Research article}

Keywords: asymmetric, refractive, diffractive, multifocal intraocular lens, visual quality

Posted Date: March 23rd, 2020

DOI: https://doi.org/10.21203/rs.2.14898/v2

License: (9) (i) This work is licensed under a Creative Commons Attribution 4.0 International License. Read Full License 


\section{Abstract}

BACKGROUND: To compare the visual performance of asymmetric refractive multifocal intraocular lenses (MIOLs) with all optic zone diffractive MIOLs.

METHODS: A prospective study. Patients underwent phacoemulsification were divided into two groups according to the type of MIOLs: 25 patients were implanted with asymmetric refractive MIOLs and 25 patients with all optic zone diffractive MIOLs. Visual acuity, refraction, defocus curves, objective optical quality and the questionnaire of life quality were measured 3 months after surgery.

RESULTS: There was no significant difference between two groups in uncorrected distance visual acuity uncorrected near visual acuity冋best corrected distance visual acuity or distance corrected near visual acuity. However, the uncorrected intermediate visual acuity was $0.24 \pm 0.10$ in refractive group and $0.31 \pm 0.13$ in diffractive group $(P<0.05)$; the distance corrected intermediate visual acuity was $0.22 \pm 0.09$ in refractive group and $0.31 \pm 0.14$ in diffractive group $(P<0.05)$. Defocus curves showed two peaks of maximum vision in both groups. However, the curve between two peaks of refractive group was smoother than diffractive group. The Modulated transfer function cut-off frequency was $₫ 22.74 \pm 12.29 \llbracket \mathrm{c} / \mathrm{d}$ in refractive group and $₫ 30.50 \pm 10.04 \llbracket \mathrm{c} / \mathrm{d}$ in diffractive group $(P<0.05)$; the OQAS values $100 \%(0 V 100 \%)$ were $0.75 \pm 0.41$ in refractive group and $1.02 \pm 0.34$ in diffractive group $\triangle \mathrm{P}<0.05 \mathbb{} \mathrm{Q} \mathrm{OV} 20 \%$ were $0.52 \pm 0.34$ in refractive group and $0.71 \pm 0.25$ in diffractive group $\otimes P<0.05 \otimes$. There was no significant difference between two groups at overall satisfaction, independence spectacles ratio or visual interference phenomenon.

CONCLUSIONS: Both MIOLs achieve good visual acuity at distance and near. The asymmetric refractive MIOLs show better intermediate visual acuity, and the all optic zone diffractive MIOLs appear to have better objective visual quality.

\section{Background}

Multifocal intraocular lenses (MIOLs) have become a widely accepted option in cataract surgery. All MIOLs developed until now with reported clinical outcomes are based on the principles of diffraction and refraction. With these technologies, incoming light rays are distributed onto near and distance focal points or onto several focal points, to provide patients good vision at all distance after cataract surgeries [1-7]. \{Alio, 2012 \#93\}Meanwhile, these designs produce several consequences such as loss of light which may cause the reduction of contrast sensitivity, permanent overlapping images generating halos and glare and loss of image quality ${ }^{[8-11]}$.

In clinic, refractive and diffractive MIOLs have proved their own advantages and side effects ${ }^{[2,4]}$. However, few studies have been done to compare these two types of MIOLs. Some researchers believe that for refractive MIOL, after eliminating the diffraction ring, the energy loss of light will be less, which is more beneficial to the improvement of visual quality ${ }^{[12-14]}$. And according to the newest design theory of refractive MIOLs, excellent objective and subjective visual acuity are able to be gained ${ }^{[2,3,15-17]}$. 
The aim of this study is to compare the visual quality of the refractive rotationally asymmetrical MIOLs with the full optic zone diffractive MIOLs using both objective and subjective methods, and to provide a comprehensive reference for personal MIOLs choice in clinic.

\section{Methods}

This non-randomized, observational study was performed at the Eye Hospital of Wenzhou Medical University (Zhejiang, China), approved by the institutional review board and ethics committee of Wenzhou Medical University. Practices and research were conducted in accordance with the Declaration of Helsinki. All patients signed informed consent before participation.

Patients' records between October 2016 to August 2017 were evaluated and fifty patients (50 eyes) with phacoemulsification and IOL implantation surgery were included. Twenty-five patients were implanted with refractive rotationally asymmetrical MIOLs (Oculentis MF30) and twenty-five patients were implanted with all optic zone diffractive MIOL (TecnisZMB00). Inclusion criteria were patients older than 50 year of age with age-related cataract, axial length between 21 and $25 \mathrm{~mm}$ and IOL was implanted in the capsular bag. Exclusion criteria were patients less than 50 years of age; with corneal astigmatism of more than $1.0 \mathrm{D}$; with cornea lesions or scars, macular diseases, ocular inflammation or any other ocular diseases; with any ocular surgical history. All patients provided informed consent.

First operated eye was selected in the study when the patient underwent binocular cataract surgeries. Visual functional data was collected by clinical researchers who did not know the IOL type the patient received.

\section{Patient assessment}

All patients had a full pre- and post-operative ophthalmologic assessment. The preoperative examination included refraction, uncorrected distance visual acuity (UDVA), best-corrected distance visual acuity (BCDVA), slit-lamp examination (SL115;Carl Zeiss, Oberkochen, Germany), fundus examination under dilation, corneal topography (Pentacam, Oculus, Inc.), endothelial cell count (SP 2000P specular microscope, Topcon Europe BV), biometry (IOLMaster, Carl Zeiss Meditec AG), retinal optical coherence tomography (Cirrus 4000 OCT, Carl Zeiss MeditecAG). Patients were evaluated at 1 day, 1 week, and 1 and 3 months. At each follow-up visit, the UDVA and BCDVA, Uncorrected near visual acuity (UNVA) and distance corrected near visual acuity (DCNVA), Uncorrected intermediate visual acuity (UIVA) and distance corrected intermediate visual acuity (DCIVA), refraction, Optical Quality Analysis System (OQAS) II were measured At the 3-month evaluation, defocusing curve was measured and patients were requested to complete a purpose-developed satisfaction questionnaire.

\section{Intraocular lens}

The Lentis Mplus LS-313 MF30 (Oculentis GmbH) (figure 1a) is an asymmetric refractive MIOL that has been extensively used. This refractive MIOL is designed with a $6.0 \mathrm{~mm}$ optic and a sector-shaped 3.0 
diopter (D) near segment. The special principle of this MIOL is that the light is not refracted symmetrically around the optical axis. The ZMB00 (Advanced Medical Optics, Santa Ana, California,USA) (figure 1b) is a diffractive MIOL with 4.0 diopter (D) near segment. It has a full diffractive optic in posterior surface with a special margin design that is round in the front portion and square in the back. Optical analog diagrams stimulate the ray tracing of these two MIOLs and show that the MF30 model has a long and narrow blur spot s (figure 2a), while the ZMB00 model has a relatively short and wide blur spot(figure 2b), which indicates the MF30 model may has an extended focal depth than the ZMB00 model.

\section{Surgical procedures}

All cataract surgeries were done by an experienced surgeon. The surgical procedure was the same for both lenses. All surgeries was performed under topical anaesthesia, using $2.2 \mathrm{~mm}$ transparent corneal incision. Phacoemulsification was performed using Infiniti vision system unit (Alcon Laboratories, Inc.). Polishing of the posterior capsule was also performed. The IOL was implanted in the capsular bag.

\section{Outcome measurements}

UDVA and BCDVA of each selected eye was measured at $5 \mathrm{~m}$ using standard logarithmic visual acuity chart with illumination of $80 \mathrm{~cd} / \mathrm{m} 2$. UNVA and DCNVA were measured the reading distance of $33 \mathrm{~cm}$ using near logarithmic visual acuity chart. UIVA and DCIVA were measured at distance of $80 \mathrm{~cm}$ using logarithmic visual acuity chart. Both intermediate and near visual acuity were measured under photopic conditions with illumination of $80 \mathrm{~cd} / \mathrm{m} 2$. Visual acuity results were recorded in logMAR.

Optical Quality Analysis System (OQAS) II is a system based on a double-pass technique, analyzing the visual quality in human eyes. OQAS analyzes all optical information of a surface, taking into account the influence of scattering, aberration and diffraction, and obtains the correct PSF image. It is the only available system to analyze the visual quality objectively at present. ${ }^{[18,19]}$. In this study, OQAS II was used to obtain the ocular objective optical quality parameters including modulation transfer function (MTF) cut-off frequency, objective scatter index (OSI), Strehl ratio (SR), and OQAS values (OVs $100 \%, 20 \%$ and 9\%) 3 months after IOL implantation. MTF is the ratio of contrast between the retinal image and the original objective. The MTF cut off indicates the The corresponding spatial frequency when MTF value is 0.01 . Normal value is larger than 30 , the larger the better. ${ }^{[20]}$. The $0 \mathrm{~V} 100 \%, 0 \mathrm{~V} 20 \%$, and $0 \mathrm{~V} 9 \%$ mean the OQAS value calculated by the system at three contrasts which are used in ophthalmic practice commonly. Therefore, these three OVs are related to the MTF curve closely. SR describes the area under the MTF curve reflects the sum of low, medium and high frequency information. Value range is $0 \sim 1$. The closer it is to 1, indicates the smaller the aberration. OSI is an index of intraocular scattered light. ${ }^{[19,21]}$. The higher the value of the MTF cut off, SR and OVs, and the lower the OSI, indicate the better the objective optical quality. The instrument was set to measure with the artificial pupil diameter of $4.0 \mathrm{~mm}$, which is a standard size used in clinical double-pass studies ${ }^{[22]}$. 
Defocus curves were used to analyze the correlations between logMAR visual acuity and image quality

measures for the two MIOLs ${ }^{[23,24]}$. It was evaluated at $5 \mathrm{~m}$ using standard logarithmic visual acuity chart. Defocus was performed from 1.0 D to $-4.0 \mathrm{D}$ in 0.5 diopter (D) steps, and started from the best correction at distance. ${ }^{[25]}$.

The subjective visual quality of post-surgery patients was investigated by using a Chinese version of the National Eye Institute Visual Function Questionnaire (CHI-VFQ-25) ${ }^{[26]}$, including subjective visual evaluation, independence spectacles ratio, and visual disturbance symptoms. Patients' subjective visual quality was evaluated with $0 \sim 10$ points, with 10 points being full marks. Whether it is necessary to wear frame glasses or corneal contact lenses at distance, medium and near after surgery would be recorded to calculate the rate of independence from spectacles. Symptoms of visual interference include glare and halo would also be asked.

\section{Data analysis}

Statistical analysis was performed using SPSS software for Windows (version 19.0, SPSS Inc.). $\chi^{2}$ test and Spearman rank correlation was used in categorical data; $t$ test and Pearson correlation were used in measurement data; Mann-Whitney $U$ test and Spearman correlation were used when a normal distribution was not expected. Results are presented as the mean $\pm S D$. Differences with $p<0.05$ were considered to be statistically significant.

\section{Results}

\section{General data}

All patients completed examinations at 3 months after cataract surgery. No intraoperative or postoperative complications occurred during the study. The preoperative conditions of patients in these two groups were analyzed including age, gender and pre-surgical VA. No significant differences were found as shown in table 1.

Table 1 Clinical and demographic data of patients implanted with refractive and diffractive MIOL (visual acuity results were recorded in $\log M A R$ )

\section{Visual and refractive outcomes}

The postoperative visual and refractive outcomes were summarized in table 2 . No statistically significant differences were found between the two groups in UDVA匹UNVA]BCDVA or DCNVA. However, the UIVA was $0.24 \pm 0.10$ in refractive group and $0.31 \pm 0.13$ in diffractive group $(P<0.05)$; the DCIVA was $0.22 \pm 0.09$ in refractive group and $0.31 \pm 0.14$ in diffractive group $(P<0.05)$ at 3 months postoperatively. Significantly better UIVA and DCIVA were found in the refractive group (table 2).

Table 2 Postoperative visual and refractive outcomes in the refractive and diffractive groups at 3 months postoperatively (results were recorded in logMAR) 


\section{Objective optical quality outcomes}

No significant differences were found in the OSI, SR, or the OV $9 \%$ between the two groups. The MTF cutoff frequency was $₫ 22.74 \pm 12.29 \rrbracket \mathrm{c} / \mathrm{d}$ in refractive group and $\mathbb{} 30.50 \pm 10.04 \rrbracket \mathrm{c} / \mathrm{d}$ in diffractive group $(P<0.05)$. The OV100\% were $0.75 \pm 0.41$ in refractive group and $1.02 \pm 0.34$ in diffractive group $(P<0.05)$, OV20\% were $0.52 \pm 0.34$ in refractive group and $0.71 \pm 0.25$ in diffractive group $(P<0.05)$. MTF cut-off frequency, OV100\% and OV20\% were significantly better in diffractive group (table 3 ).

Table 3 Objective measures by OQAS II in the refractive and diffractive groups at 3 months postoperatively (the pupil is $4 \mathrm{~mm}$ )

\section{Defocusing curve}

As shown in the defocusing curve, both groups provide two peaks of maximum vision. For refractive group, one peak at OD defocus level which corresponds to the BCDVA and the second peak at -3.0 D defocus level which corresponds to the DCNVA. For diffractive group, one peak at $-0.5 \mathrm{D}$ defocus level which corresponds to the BCDVA and the second peak at -3.0 D defocus level which corresponds to the DCNVA.

However, the curve between two peaks of refractive group was smoother than diffractive group which indicates a better intermediate visual acuity (Figure 3).

\section{VF questionnaire}

In the refractive MIOL group, 7 patients (28\%) developed glare and halo after surgery, while 6 patients (24\%) in the diffractive MIOL group. There was no statistically significant difference between the two groups $(P>0.05)$. There also no significant differences were found in the independence spectacles ratio or overall satisfaction between the two groups $(P>0.05$, table 4$)$.

Table 4 Questionnaire results in the refractive and diffractive groups at 3 months postoperatively

\section{Discussion}

Refractive and diffractive MIOLs are two main types MIOLs popularly used in cataract surgery. In this study, we found the improvement of UDVA and BCDVA in both groups to be good and similar, which is consistent with previous findings reported. These two MIOLs seem to have a similar ability to successfully restore the distance visual function after cataract surgery. Also, results showed both groups gained good UNVA and DCNVA, which means both MIOLs are able to provide enough near visual function at reading distance for post-surgery patients. And there results are also consistent with previous reports. $[2,4,5,8,17]$. However, better results were found in UIVA and DCIVA for the refractive group. Also, defocus curves showed a smoother curve in the refractive group than the diffractive group, which was consistent with the visual outcomes. These outcomes were expected. According to the design theory, the refractive MIOL model is based on the concept of refractive rotational asymmetry with an inferior surface- 
embedded segment. The segment with the optical power is required for near vision and seamless transitions between the near and far vision zones. This design theoretically allows this MIOL to be independent of pupil size and ensures optimal adjustment of near and distance vision. Therefore, we believe this refractive MIOL model should provide better intermediate vision performance. However, there is one limit we should take into account. The refractive MIOL has a +3.0 near additional (ADD) power, and the diffractive MIOL has a +4.0 near ADD power. The defocus curve showed a smoother transition between the 2 peaks in the refractive MIOL than the diffractive MIOL, and the different performance of intermediate vision may also be attributed to the different near ADD power.

In the study, we found MTF cut-off frequency, OV100\% and OV20\% were significantly better in the diffractive group under $4 \mathrm{~mm}$ pupil than the refractive group, which was not consistent with some previous studies. ${ }^{[19,21,22]}$. In our study, all outcomes derived from OQASII of the diffractive group were better than the outcomes of the refractive group as seen in table 3. Moreover, the differences of MTF cutoff frequency, OV100\% and OV20\% between two groups were statistically significant. Although the differences of OSI, SR and OV9\% between two groups were not significant, which we considered may because of the limited case numbers. Therefore, it seems that the all optic zone diffractive MIOLs appear to have better objective visual quality than the asymmetric refractive MIOLs. In our study, only patients with pupil diameter no less than $4 \mathrm{~mm}$ were allowed to be measured. And we also have checked its repeatability before we started this study. Therefore, we believe this subjective measurement can been used to evaluate the MIOLs in our study and the results is reliable. Besides, the OQAS results of the diffractive MIOL in this study is very close to the results we gained in a previous report ${ }^{[5]}$, which also indicates that the data is reliable.

Meanwhile, no significant differences were found in subjective visual quality between the two groups. We found no difference in glare, halo, and independence from spectacles or patient satisfaction between the two groups. Besides, previous studies have report that ZMB00 has similar subjective visual quality with same material monofocal IOL ${ }^{[1,5]}$.

These findings indicate that the MF30 MIOL does not seem to provide a good subjective or objective visual quality, although it can provide a good intermediate vision. Meanwhile, the ZMBOO IOL appears to provide even better objective visual quality. We consider that for the refractive MIOL, although giving up using diffractive rings in optical zone may be beneficial to the use of light rays, however, the division of the pupil area could result in loss of light source, which may cause the impairment of objective and subjective visual quality. For the diffractive MIOL ZMBO0, the material properties and the design of MIOL may play important roles in improvement of visual quality. Recent studies have shown that ability of achromatisation by diffractive MIOLs could provide significant improvement in polychromatic retinal image quality ${ }^{[5,27]}$. The diffractive MIOL model is with a high Abbe number of 55.5 . Therefore, contrast sensitivity increased when compared with other materials may due to the lower chromatic dispersion, which could minimize the impact of chromatic aberration. Besides, the ZMB00 model uses posterior diffractive surface to concentrate light instead of anterior surface, which may also help in better focus and improving visual performance. In addition, compared with the traditional reticular margin design, 
ZMB00 model has a special margin design with round margin in front and square in the back. This type of design may help in reducing the diffraction and refraction along the edge. We believe all these factors may contribute to the improvement of optical quality.

\section{Conclusions}

In conclusion, we found that both MIOL models are able to provide good visual acuity at distance and near. The asymmetric refractive MIOLs show better intermediate visual acuity, and the all optic zone diffractive MIOLs appear to have better objective visual quality. These results suggest that apart from paying attention to the improvement of the principle of focal separation, we should also pay attention to aberrations, color differences, IOL edge design and the placement of the front and back of the focal plane. In further studies, we should analyze a larger sample of patients over a long-term follow-up.

\section{Abbreviations}

MIOLs, multifocal intraocular lenses; UDVA, uncorrected distance visual acuity; BCDVA, best-corrected distance visual acuity; DCNVA, distance corrected near visual acuity; UIVA, uncorrected intermediate visual acuity; DCIVA, distance corrected intermediate visual acuity; OQAS, optical Quality Analysis System.

\section{Declarations}

1. Ethics approval and consent to participate: our study was submitted to and approved by the institutional ethics committee of Eye Hospital of Wenzhou Medical University. This study was obtained written informed consent from all participants.

2. Consent for publication: This study was obtained written informed consent from all participants.

3. Availability of data and materials: Data and materials of this analysis were extracted from the original researches which were referenced in this article.

4. Competing interests: The authors declare no relevant conflict of interest.

5. Funding: There is no funding for this work.

6. Authors' contributions: The authors on this paper all participated in study design. All authors read, critiqued and approved the manuscript revisions as well as the final version of the manuscript. Also, all authors participated in a session to discuss the results and consider strategies for analysis and interpretation of the data before the final data analysis was performed and the manuscript written.

7. Acknowledgements: Not Applicable.

\section{Funding}

National Natural Science Foundation of China (Grant No 81670784).

\section{Competing interests}


The authors declare no interest.

\section{References}

1. Yamauchi, T., et al., Comparison of visual performance of multifocal intraocular lenses with same material monofocal intraocular lenses. PLoS One, 2013. 8(6): p. e68236.

2. Alio, J.L., et al., Comparison of the visual and intraocular optical performance of a refractive multifocal IOL with rotational asymmetry and an apodized diffractive multifocal IOL. J Refract Surg, 2012. 28(2): p. 100-5.

3. Alfonso, J.F., et al., Visual function comparison of 2 aspheric multifocal intraocular lenses. J Cataract Refract Surg, 2012. 38(2): p. 242-8.

4. Gatinel, D. and Y. Houbrechts, Comparison of bifocal and trifocal diffractive and refractive intraocular lenses using an optical bench. J Cataract Refract Surg, 2013. 39(7): p. 1093-9.

5. Chen, T., et al., Objective and subjective visual quality after implantation of all optic zone diffractive multifocal intraocular lenses: a prospective, case-control observational study. Br J Ophthalmol, 2016. 100(11): p. 1530-1535.

6. Ruiz-Mesa, R., et al., Comparison of visual outcomes after bilateral implantation of extended range of vision and trifocal intraocular lenses. European Journal of Ophthalmology. 27(4): p. 460-465.

7. Mcneely, R.N., et al., Visual quality and performance comparison between 2 refractive rotationally asymmetric multifocal intraocular lenses. Journal of Cataract \& Refractive Surgery. 43(8): p. 10201026.

8. Alio, J., et al., Multifocal intraocular lenses: An overview. Surv Ophthalmol, 2017. 62(5): p. 611-634.

9. de Vries, N.E. and R.M. Nuijts, Multifocal intraocular lenses in cataract surgery: literature review of benefits and side effects. Journal of Cataract \& Refractive Surgery, 2013. 39(2): p. 268-278.

10. Javitt, J., et al., Cataract extraction with multifocal intraocular lens implantation: clinical, functional, and quality-of-life outcomes. Multicenter clinical trial in Germany and Austria. J Cataract Refract Surg, 2000. 26(9): p. 1356-1366.

11. T, A.H. and H. HA, Spectacle Independence And Patient Satisfaction With Pseudophakic MiniMonovision Using Aberration-Free Intraocular Lens. Clinical ophthalmology (Auckland, N.Z.), 2019. 13(undefined): p. 2111-2117.

12. Bala, C. and K. Meades, Improvement in vision with inverted placement of an asymmetric refractive multifocal intraocular lens. J Cataract Refract Surg, 2014. 40(5): p. 833-5.

13. Yu, S., et al., Clinical Outcomes of Patients with Refractive Aspheric Multifocal IOL Implantation. Journal of the Korean Ophthalmological Society, 2014. 55(7): p. 991.

14. Tchah, H., K. Nam, and A. Yoo, Predictive factors for photic phenomena after refractive, rotationally asymmetric, multifocal intraocular lens implantation. Int J Ophthalmol, 2017. 10(2): p. 241-245.

15. Moore, J.E., et al., Rotationally asymmetric multifocal intraocular lenses: preoperative considerations and postoperative outcomes. Curr Opin Ophthalmol, 2017. 28(1): p. 9-15. 
16. McAlinden, C. and J.E. Moore, Multifocal intraocular lens with a surface-embedded near section: Short-term clinical outcomes. Journal of Cataract \& Refractive Surgery, 2011. 37(3): p. 441-445.

17. Venter, J.A., et al., Visual outcomes and patient satisfaction with a rotational asymmetric refractive intraocular lens for emmetropic presbyopia. J Cataract Refract Surg, 2015. 41(3): p. 585-93.

18. Bueno, J.M., et al., Impact of scatter on double-pass image quality and contrast sensitivity measured with a single instrument. Biomed Opt Express, 2015. 6(12): p. 4841-9.

19. Pan, A.P., et al., Correlation among lens opacities classification system III grading, visual function index-14, pentacam nucleus staging, and objective scatter index for cataract assessment. Am J Ophthalmol, 2015. 159(2): p. 241-7 e2.

20. Saad, A., M. Saab, and D. Gatinel, Repeatability of measurements with a double-pass system. Journal of Cataract \& Refractive Surgery, 2010. 36(1): p. 28-33.

21. Xu, C.C., et al., Repeatability and reproducibility of a double-pass optical quality analysis device. PLoS One, 2015. 10(2): p. e0117587.

22. Meritxell, V., et al., Optical quality of foldable monofocal intraocular lenses before and after injection: comparative evaluation using a double-pass system. Journal of Cataract \& Refractive Surgery, 2009. 35(8): p. 1415-1423.

23. Buckhurst, P.J., et al., Multifocal intraocular lens differentiation using defocus curves. Invest Ophthalmol Vis Sci, 2012. 53(7): p. 3920-6.

24. Gil, M.A., et al., Visual acuity and defocus curves with six multifocal intraocular lenses. International Ophthalmology, 2019(2).

25. S, P., et al., Comparison of visual acuities at different distances and defocus curves. Journal of cataract and refractive surgery, 2002. 28(11): p. 1964-7.

26. Chan, C.W.S., et al., Development of a Chinese version of the National Eye Institute Visual Function Questionnaire (CHI-VFQ-25) as a tool to study patients with eye diseases in Hong Kong. $\mathrm{Br} \mathrm{J}$ Ophthalmol, 2009. 93(11): p. 1431-1436.

27. Lubinski, W., J. Gronkowska-Serafin, and K. Podboraczynska-Jodko, Clinical outcomes after cataract surgery with implantation of the Tecnis ZMBO0 multifocal intraocular lens. Med Sci Monit, 2014. 20: p. 1220-6.

\section{Tables}

Table 1 Clinical and demographic data of patients implanted with refractive and diffractive MIOL (visual acuity results were recorded in logMAR)

\begin{tabular}{llll}
\hline & Refractive MIOL & Diffractive MIOL & p Value \\
\hline Age & $69.48 \pm 8.09$ & $68.41 \pm 8.77$ & 0.331 \\
Gender(male/female) & $15 / 10$ & $13 / 12$ & 0.189 \\
Presurgical VA & $0.51 \pm 0.22$ & $0.51 \pm 0.21$ & 0.478 \\
\hline
\end{tabular}


Data are expressed as the mean $\pm S D$ or median (range). The difference between groups was analyzed by independent $t$ test for data in accordance with normal distribution. The Mann-Whitney $U$ test was used for data that did not conform to normal distribution.

Categorical data was compared using $\chi^{2}$ test.

MIOL, multifocal intraocular lens; VA, visual acuity.

Table 2 Postoperative Visual and Refractive Outcomes in the refractive and diffractive groups at 3 months postoperatively (results were recorded in logMAR)

\begin{tabular}{llll}
\hline & Refractive MIOL & Diffractive MIOL & p Value \\
\hline Distance & & & \\
Uncorrected & $0.06 \pm 0.07$ & $0.09 \pm 0.09$ & 0.236 \\
Best corrected & $0.05 \pm 0.06$ & $0.03 \pm 0.05$ & 0.337 \\
Intermediate & & & \\
Uncorrected & $0.24 \pm 0.10$ & $0.32 \pm 0.13$ & $0.029^{*}$ \\
Distance corrected & $0.22 \pm 0.09$ & $0.32 \pm 0.14$ & $0.016^{*}$ \\
Near & & & \\
Uncorrected & $0.23 \pm 0.12$ & $0.24 \pm 0.14$ & 0.800 \\
Distance corrected & $0.21 \pm 0.11$ & $0.20 \pm 0.12$ & 0.866 \\
\hline
\end{tabular}

Data are expressed as the mean $\pm S D$ or median (range). The difference between groups was analyzed by independent $t$ test for data in accordance with normal distribution. The Mann-Whitney $U$ test was used for data that did not conform to normal distribution.

*Statistically significant difference between groups ( $p$ value $<0.05$ ).

Table 3 Objective measures by OQAS II in the refractive and diffractive groups at 3 months postoperatively (the pupil is $4 \mathrm{~mm}$ )

\begin{tabular}{llll}
\hline & Refractive MIOL & Diffractive MIOL & p Value \\
\hline MTF cut-off (cpd) & $22.74 \pm 12.30$ & $30.18 \pm 9.81$ & $0.014^{\star}$ \\
OSI & $2.52 \pm 1.28$ & $2.16 \pm 0.98$ & 0.294 \\
Strehl ratio & $0.14 \pm 0.08$ & $0.17 \pm 0.06$ & 0.104 \\
OV100\% & $0.75 \pm 0.41$ & $1.05 \pm 0.33$ & $0.011^{\star}$ \\
OV20\% & $0.52 \pm 0.34$ & $0.72 \pm 0.25$ & $0.030^{\star}$ \\
OV9\% & $0.33 \pm 0.23$ & $0.42 \pm 0.15$ & 0.100 \\
\hline
\end{tabular}

Data are expressed as the mean $\pm S D$ or median (range). The difference between groups was analyzed by independent t test for data in accordance with normal distribution. The Mann-Whitney $U$ test was used for data that did not conform to normal distribution.

*Statistically significant difference between groups ( $p$ value $<0.05$ ).

OQAS, optical quality analysis system; MTF, modulation transfer function; OSI, objective scatter index; SR, Strehl ratio; OV, OQAS value. 
Table 4 Questionnaire results in the refractive and diffractive groups at 3 months postoperatively

\begin{tabular}{llll}
\hline & Refractive MIOL & Diffractive MIOL & p Value \\
\hline Glare/halo & $7 / 25$ & $6 / 25$ & 0.784 \\
Independence spectacles ratio & $15 / 25$ & $20 / 25$ & 0.240 \\
overall satisfaction(score $\geq 9$ ) & $14 / 25$ & $15 / 25$ & 0.971 \\
\hline
\end{tabular}

Data are expressed as the mean $\pm S D$ or median (range). The difference between groups was analyzed by $\chi 2$ test and Spearman rank correlation.

\section{Figures}

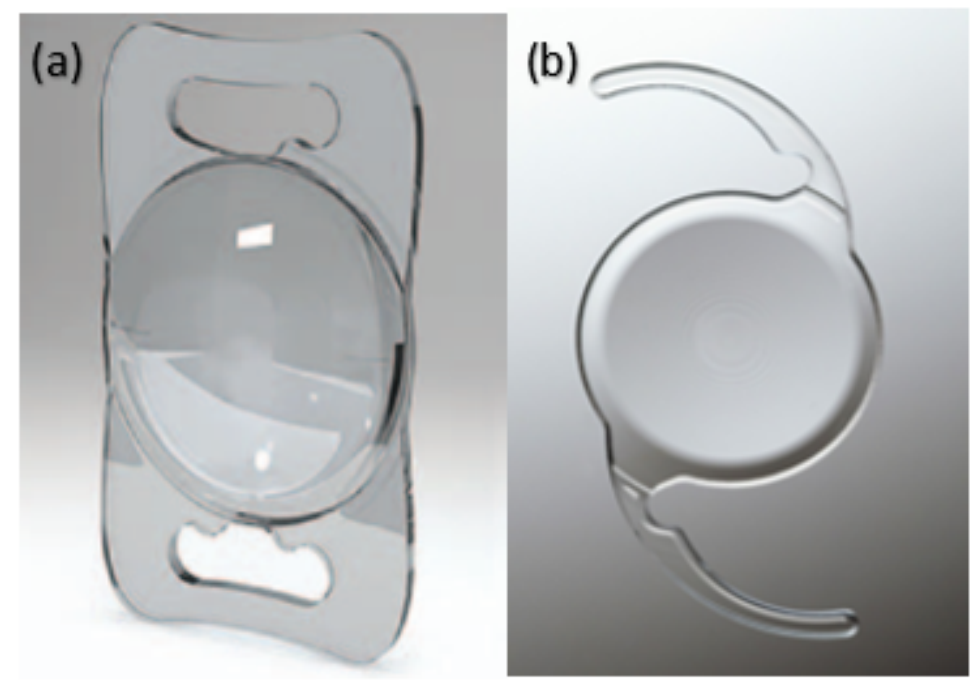

Figure 1

(a) Lentis Mplus LS-313 MF30 multifocal IOL (b) ZMB00 multifocal IOL
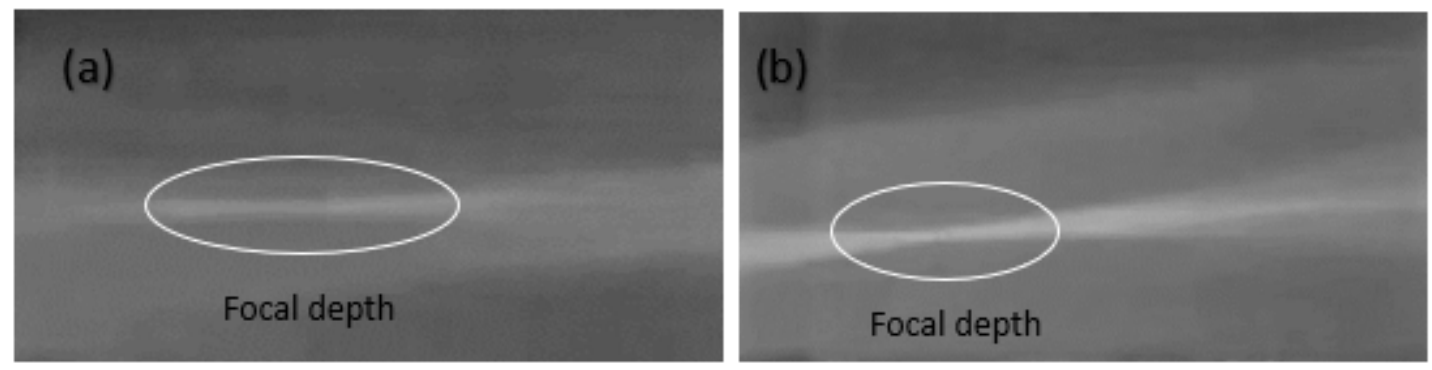

\section{Figure 2}

Ray tracing diagrams show the focal depth of each multifocal IOL. (a) Lentis Mplus LS-313 MF30 multifocal IOL (b) ZMBO0 multifocal IOL 


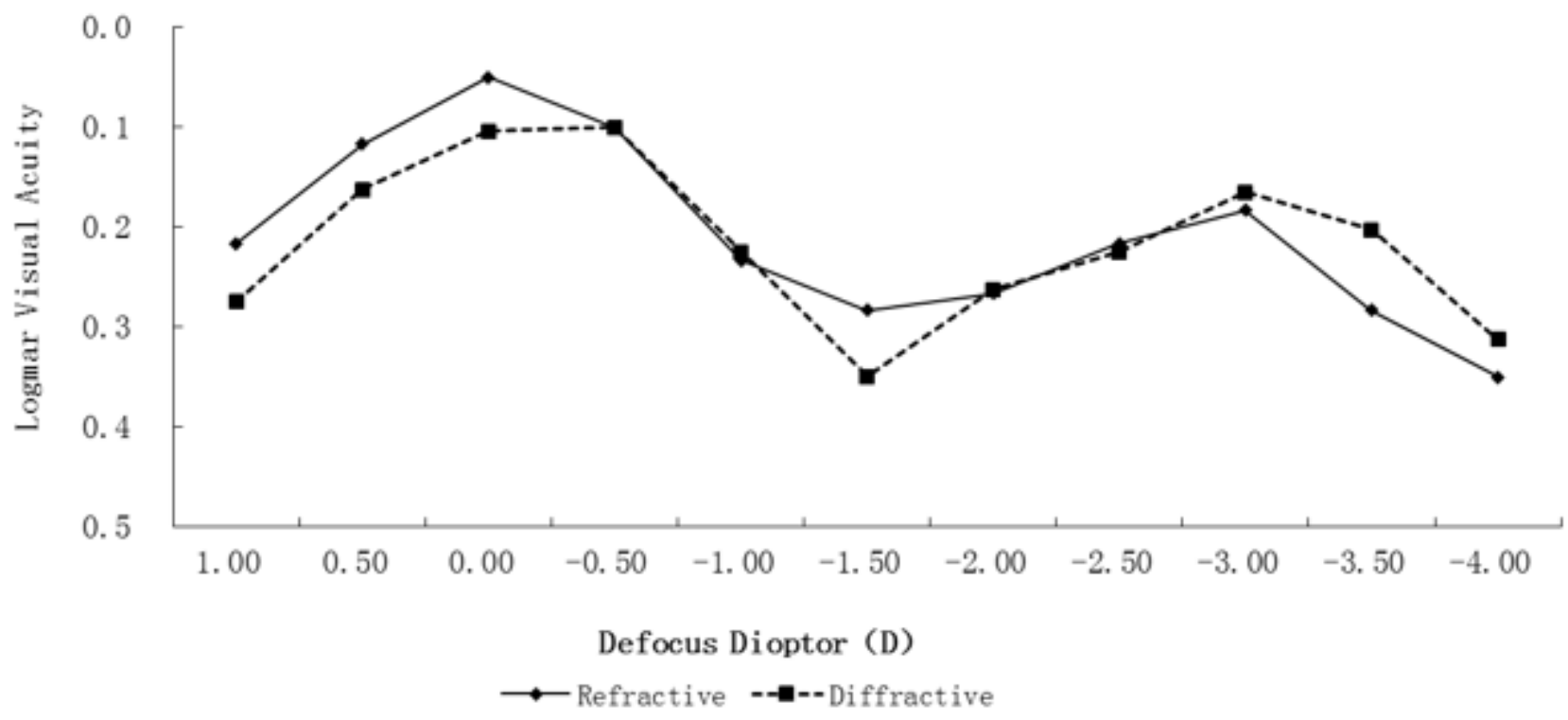

Figure 3

Defocusing curves of refractive and diffractive groups

\section{Supplementary Files}

This is a list of supplementary files associated with this preprint. Click to download.

- STROBEStatement.pdf 